\title{
GYNECOLOGICAL AND OBSTETRICAL ASPECTS OF ENTEROBIUS VERMICULARIS INFECTION
}

\author{
BALÁZS DEZSÉNYI $^{1 *}$, LÁSZLÓ SÁRKÖZI ${ }^{2}$, LÁSZLÓ KAISER ${ }^{3}$, KLÁRA TÁRKÁNYI $^{4}$, \\ RADKA NIKOLOVA ${ }^{4}$ and ZORÁN BELICS ${ }^{2}$ \\ ${ }^{1}$ Department of Infectology, Joint Hospital of Saint Stephen and Saint Ladislau, \\ Budapest, Hungary \\ ${ }^{2}$ Department of Gynecology and Obstetrics, Jahn Ferenc South Pest Hospital, \\ Budapest, Hungary \\ ${ }^{3}$ Department of Pathology, Jahn Ferenc South Pest Hospital, Budapest, Hungary \\ ${ }^{4}$ Laboratory of Microbiology, Joint Hospital of Saint Stephen and Saint Ladislau, \\ Budapest, Hungary
}

(Received: 20 January 2018; accepted: 10 February 2018)

\begin{abstract}
Enterobiasis is an easily recognizable and treatable disease with higher incidence among children. Based on these facts, its clinical importance is usually underestimated. This infection also occurs during adulthood and without treatment, it can cause severe complications in some rare cases. Unnoticed subclinical infection in women can lead to infertility and even to life-threatening conditions. It is also emphasized in this paper that the treatment of identified enterobiasis during pregnancy needs caution. After reviewing the current scientific literature, two gynecological cases are presented here.
\end{abstract}

Keywords: enterobiasis gravidorum, mebendazole during pregnancy

\section{Introduction}

Human is the only known host of Enterobius vermicularis (pinworm) with infection totaling over 200 million worldwide, making it the most prevalent human helminthosis. Thirty percentage of children are infected with this parasite favoring the 7- to 11-year group; female to male infectivity is 3:1 [1]. Geographically, the parasite is more commonly seen in temperate climates. Factors such as overcrowding and poor sanitation increase infectivity [2]. The infective cycle begins with ingestion of ova transferred from the perianal skin with contaminated finger or tools. After the hatching of larvae, they mature during their migration to the mating site in the cecum and its vicinity. After fertilization, females migrate to the

\footnotetext{
*Corresponding author; E-mail: dezsenyibalazs13@gmail.com
} 
perianal region to deposit eggs. This leads to symptoms of perianal pruritus and scratching especially during night time, which may result in finger contamination, thus completing the cycle. Infection can be transmitted $a b$ ano $a d$ os as autoinfection and directly from person-to-person $[1,2]$. In Hungary, there is no official data about the prevalence of this infection as it does not belong to the reportable helminthic infections [edict VI.3./18/1998 of Hungarian Ministry of Social Welfare (Népjóléti Minisztérium, NM)]. Microscopic identification of eggs in a sample collected from the perianal area by a transparent adhesive tape is the method of choice for diagnosing enterobiasis. The medications used for the treatment of pinworm are mebendazole, pyrantel pamoate, or albendazole [3]. In Hungary, benzimidazole derivatives, such as mebendazole and albendazole, are currently available. Data on the use of benzimidazoles in pregnant woman are scarce. A prospective-controlled cohort study that followed 192 pregnant women treated with mebendazole suggested that mebendazole does not represent a major teratogenic risk in humans when it is used in the doses that are commonly used for pinworm (E. vermicularis) infestation [4]. Benzimidazoles are in pregnancy category $\mathrm{C}$, which means that studies in animals have revealed adverse effects on the fetus (teratogenic or embryocidal, or other) and there are just limited number of controlled studies in women or studies in women and animals are not available. Drugs should be given only in the second and third trimesters, if the potential benefit justifies the potential risk to the fetus [3].

\section{Case 1}

A 46-year-old female patient [gravidity, 4 (G:4); parity, 4 (P:4)] at the middle of her menstrual cycle was admitted to the Accident and Emergency Department of Jahn Ferenc South Pest Hospital on December 12, 2016. The afebrile patient complained about sudden onset abdominal pain, nausea, and difficulties on starting urination. On physical examination, only mild suprapubic tenderness was found without muscular defense or palpable resistance. Results of laboratory investigations showed inflammation [white blood cells: $19 \mathrm{G} / \mathrm{L}$; C-reactive protein (CRP): $135 \mathrm{mg} / \mathrm{L}$ ] and normal kidney function (creatinine: $68 \mathrm{umol} / \mathrm{L}$; blood urea nitrogen: $2.2 \mathrm{mmol} / \mathrm{L}$ ). Urine analysis revealed neither pyuria nor hematuria. Urological examination was also performed but did not suggest any urological causes as the origin of her symptoms. Abdominal and pelvic ultrasound examination revealed small cystic changes in the right ovary with normal left ovary and urinary tract morphology. Although the patient was offered inpatient care, she left the hospital against medical advice. One day later, the patient was seen again in the Accident and Emergency Department of Jahn 
Ferenc South Pest Hospital with persisting and intensifying left abdominal pain. On physical examination, severe pain was detected in the suprapubic area with defense musculaire. Laboratory results showed worsening inflammation (white blood cells: 15 G/L; CRP: $211 \mathrm{mg} / \mathrm{L}$ ). Possible gynecological causes were raised during surgical consultation. Subsequent gynecological examination showed abdominal pain at the pelvis proper mainly on the left side together with cervical motion tenderness. During transvaginal ultrasound examination, bilaterally enlarged ovaries with cystic lesions and echogenic content were predominantly found at the left side suggesting inflammation and tubo-ovarian abscess. Hospital admission was carried out and antibiotic therapy was initiated using clindamycin, gentamicin parenterally, and doxycycline orally. Preoperative repeated ultrasound examination also identified a multiloculated cyst $(29 \times 24 \mathrm{~mm})$ with echogenic fluid content at the left ovary $(48 \times 26 \mathrm{~mm})$ and showed an adnexal mass at the right ovary $(63 \times 41 \mathrm{~mm})$. During laparotomy, inflamed cystic ovaries and livid and expanded fallopian tubes were explored on both sides with normal uterus. The age of the patient and the severe bilateral tubo-ovarian inflammation were considered, and finally hysterectomy and bilateral salpingo-oophorectomy were performed. Escherichia coli was cultured from the abdominal fluid and it was found to be susceptible to the empirically applied antibiotics. On macroscopic examination of the hysterectomy and salpingo-oophorectomy specimens, the serosal surface showed a dull opaque appearance with purulent exudate. The specimens were dissected according to pathological protocols. On opening the uterine cavity and on slicing the tissue, congested organs were seen. Both fallopian tubes showed dilatation and were expanded by purulent exudate. The right ovary showed enlargement while numerous smaller cysts divided the left one. Representative sections were taken according to pathological protocols sampling both normal looking areas and those showing alterations macroscopically. Histopathology confirmed the clinical and macroscopic observations and concluded the diagnosis of tubo-ovarian abscess. In addition, in one focus - from a left fallopian tube sample - elongated, asymmetrical, flattened, calcified bodies were found with smooth, thin eggshell in which C-shaped larvae were seen. They were surrounded by foreign body-type and Langhans-type giant cell reaction (Figure 1). In some cross sections, narrow, eosinophilic lateral cuticular layer and internal organs of an adult nematode could be identified. The histological appearance was consistent with the prolonged presence of an adult female E. vermicularis. About 5 days after the operation, the patient left the hospital without symptoms and further healing was uncomplicated. She was then presented at our Infectology Unit of Joint Hospital of Saint Stephen and Saint Ladislau. Subsequent questioning revealed that her daughter had been suffered from E. vermicularis infection previously. There was not any suggestion of on-going pinworm infection before the 


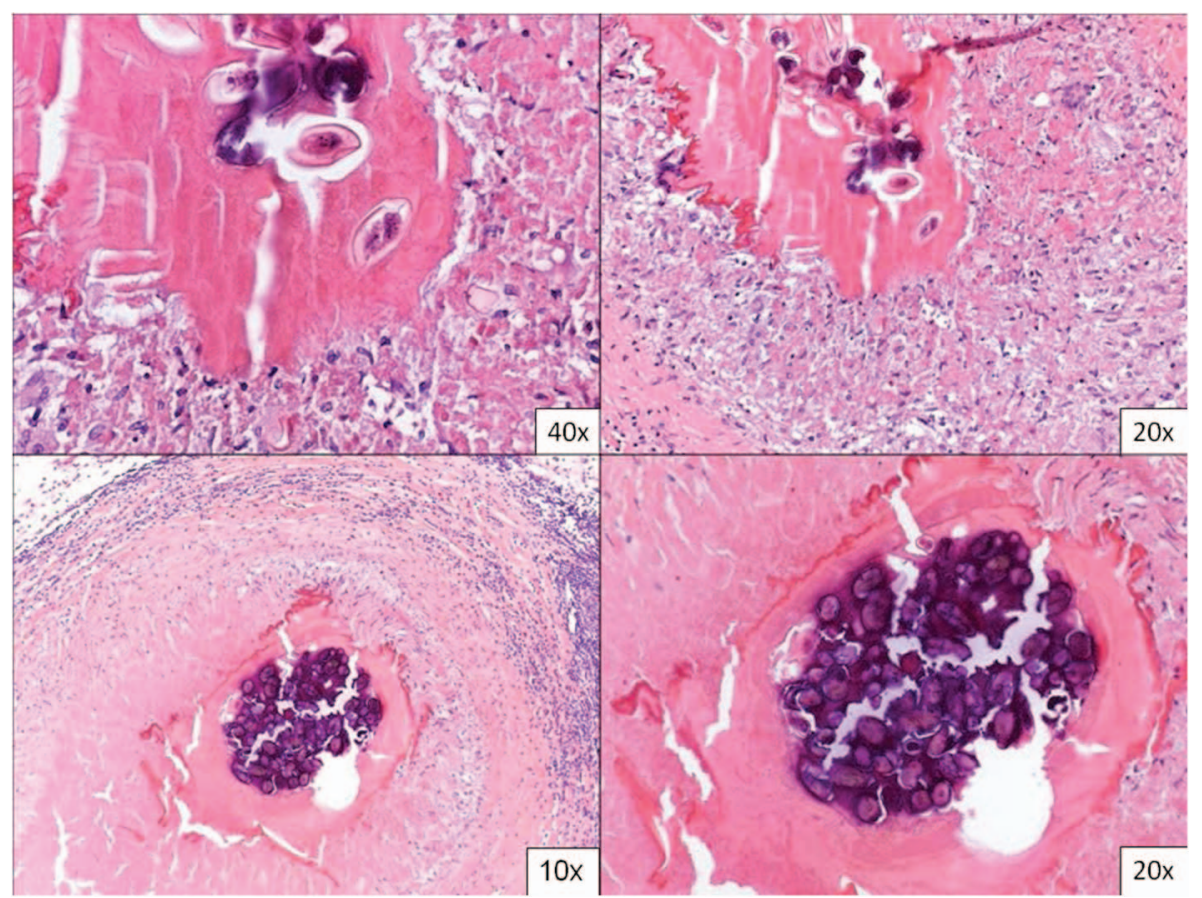

Figure 1. Sections from the fallopian tube show numerous epithelioid cell granulomas centered around ova of Enterobius vermicularis and its calcified groups (hematoxylin-eosin stain, original magnification, $10 \times, 20 \times, 40 \times)$

tubo-ovarian presentation. Following operation, the microscopic analysis of material from the perianal area collected with transparent adhesive tape confirmed the presence of ova of E. vermicularis. Patient received mebendazole and after 14 days, examination of transparent adhesive tape samples indicated effective treatment and parasitological cure.

\section{Case 2}

A 29-year-old female (G:1; P:1) patient was presented on the 18/40 weeks of her second pregnancy at our Infectology Unit of the Joint Hospital of Saint Stephen and Saint Ladislau on June 28, 2016 with the clinical symptoms of perianal pruritus. She also told that she detected one or two white, tiny worms in her stool. Previously, her daughter who had symptoms consistent with enterobiasis had been treated with mebendazole and the child became symptomless. From the medical history of the patient, microcytic anemia is worth to mention necessitating 
transfusion and iron supplementation. Her last known hemoglobin level was $101 \mathrm{~g} / \mathrm{L}$. She was continuously presented at regular gynecological check-up, and physiological conditions were studied. During the microscopic analysis of material from the perianal area collected with transparent adhesive tape, ova of E. vermicularis were detected. Given her mild symptoms and the potential teratogen effect of mebendazole, her treatment was postponed following delivery and the patient became informed about the importance of personal and environmental hygiene. On the 25/40 weeks of pregnancy, the patient and her general practitioner indicated that she became symptomless. On routine gynecological examinations, physiological conditions were found and no further worms were reported from stool by the patient. She gave birth to a healthy child but failed to attend our Infectology Unit of Joint Hospital of Saint Stephen and Saint Ladislau; therefore, no further tests could be carried out until the submission of this manuscript.

\section{Discussion}

Extraintestinal E. vermicularis has been reported in a variety of sites including most commonly the female genital tract as well as the lungs, liver, spleen, kidney, prostate, and bladder [5]. Although genital tract infections are usually asymptomatic, if symptoms occur, they reflect the site of involvement: vaginitis, endometritis, tubo-ovarian abscess, pelvic inflammatory disease, or peritonitis. Infertility or peritonitis may be a secondary complication [6-8]. The probable mechanism of spread is migration of gravid female worms from the perianal region to the vagina and subsequent ascent of parasites along the genital tract. Supporting evidence for this includes the exclusive presence of female worms and ova in reproductive tract lesions [2]. A case has been reported in the scientific literature when the parasite ascending in the fallopian tube caused salpingitis with extrauterine gravidity and led to acute abdominal catastrophe with bleeding to the abdominal cavity [9]. Thereby, enterobiasis should be treated with antihelminthic agents after its confirmation. Use of the recommended benzimidazole compounds during the first trimester of pregnancy is contraindicated, because their teratogen effect is proven in animal studies. First case reported in this paper summarizes the clinical history of a Hungarian middle-aged woman who presumably acquired enterobiasis from her child and the unnoticed, asymptomatic E. vermicularis infection was clinically presented as acute abdomen. Complication of the infection was bilateral tubo-ovarian abscess. As a consequence, uterus extirpation was performed with bilateral adnexectomy. This case justifies the urgent benzimidazole treatment of $E$. vermicularis infections in women. The second reported case supports that the careful evaluation of cases 
identified during pregnancy is crucial. In case of enterobiasis gravidorum, careful obstetrical and parasitological follow-up and postponed antihelminth treatment are reasonable choices; however, we have supporting data on the safe use of low-dose mebendazole during pregnancy [4].

Enterobiasis is a frequent infection in Hungary. In some cases, it remains unnoticed and untreated in its asymptomatic form. In women, the egg-laying of female worms ascending along the genital tract can cause severe inflammatory reaction, which can lead to infertility or even to an acute life-threatening condition. As a consequence, it is suggested to make efforts to identify the infection of E. vermicularis in women in the presence of appropriate data in the patient history. Microscopical analysis of a sample collected from the perianal area by a transparent adhesive tape is easy to perform, cheap, and ensures rapid result. In case of enterobiasis gravidorum, regular obstetrical and parasitological follow up have to be suggested, because hypothetically spontaneous clearance of $E$. vermicularis can be achieved with good patient compliance and rigorous application of certain hygienic rules. Follow-up of patients is important to provide further data to elucidate the course of infection in women and to prevent future complications.

\section{Acknowledgements}

LK performed histology and photography at Jahn Ferenc South Pest Hospital, Department of Pathology, Budapest.

\section{Conflict of Interest}

None.

\section{Patient Consent}

Obtained.

\section{References}

1. Smolyakov, R., Talalay, B., Yanai-Inbar, I., Pak, I., Alkan, M.: Enterobius vermicularis infection of female genital tract: A report of three cases and review of literature. Eur J Obstet Gynecol Reprod Biol 107, 220-222 (2003).

2. Young, C., Tataryn, I., Kowalewska-Grochowska, K. T., Balachandra, B.: Enterobius vermicularis infection of the fallopian tube in an infertile female. Pathol Res Pract 206, 405-407 (2010). 
3. www.cdc.gov/dpdx/enterobiasis/index.html

4. Diav-Citrin, O., Shechtman, S., Arnon, J., Lubart, I., Ornoy, A.: Pregnancy outcome after gestational exposure to mebendazole: A prospective controlled cohort study. Am J Obstet Gynecol 188, 282-285 (2003).

5. Podgajski, M., Kukura, V., Duic, Z., Gasparov, S., Madzarac, M.: Ascites, high CA-125 and chronic pelvic pain in an unusual clinical manifestation of Enterobius vermicularis ovarian and sigmoid colon granuloma. Eur J Gynaecol Oncol 28, 513-515 (2007).

6. Craggs, B., De Waele, E., De Vogelaere, K., Wybo, I., Laubach, M., Hoorens, A., De Waele, B.: Enterobius vermicularis infection with tuboovarian abscess and peritonitis occurring during pregnancy. Surg Infect (Larchmt) 10, 545-547 (2009).

7. Donofrio, V., Insabato, L., Mossetti, G., Boscaino, A., de Rosa, G.: Enterobius vermicularis granuloma of the ovary: Report of a case with diagnosis by intraoperative cytology. Diagn Cytopathol 11, 205-206 (1994).

8. Kogan, J., Alter, M., Price, H.: Bilateral Enterobius vermicularis salpingo-oophoritis. Postgrad Med 73, 309-310 (1983).

9. Ngui, R., Ravindran, S., Ong, D. B., Chow, T. K., Low, K. P., Nureena, Z. S., Rajoo, Y., Chin, Y. T., Amir, A., Ahmad, A. F., Lim, Y. A., Mahmud, R.: Enterobius vermicularis salpingitis seen in the setting of ectopic pregnancy in a Malaysian patient. J Clin Microbiol 52, 3468-3470 (2014). 